PROCEEDINGS OF THE

AMERICAN MATHEMATICAL SOCIETY

Volume 130, Number 4, Pages 1183-1187

S 0002-9939(01)06174-3

Article electronically published on October 1, 2001

\title{
STRONGLY MEAGER SETS OF REAL NUMBERS AND TREE FORCING NOTIONS
}

\author{
ANDRZEJ NOWIK AND TOMASZ WEISS
}

(Communicated by Carl G. Jockusch, Jr.)

Abstract. We show that every strongly meager set has the $l_{0^{-}}$and the $m_{0^{-}}$ property.

In $\left[\mathrm{NW}\right.$ it was proven that every strongly meager subset of $2^{\omega}$ is a completely Ramsey null $\left(C R_{0}\right)$ set. In this paper we show that an analogous result holds for Laver and Miller notions of forcing, i.e., every strongly meager set has both the $l_{0^{-}}$and the $m_{0}$-property. Notice that the three classes of subsets of $2^{\omega}$ mentioned above are not related as far as inclusion is concerned. This fact is due to J. Brendle (see $[\mathrm{B}]$ ).

Our terminology is standard and can be found in $\mathrm{B}$ and $\mathrm{NW}$. If $T$ is a tree on $\omega^{<\omega \uparrow}$, the set of all increasing sequences of natural numbers, then the $\operatorname{stem}(T)$ of $T$ is the unique $s \in T$ (if such $s$ exists) with $\forall_{t \in T} s \subseteq t \vee t \subseteq s$ and $\mid\{n \in \omega$ : $s \frown\langle n\rangle \in T\} \mid \geq 2$. Given $t \in T$, we define $\operatorname{succ}_{T}(t)=\{n \in \omega: t \frown\langle n\rangle \in T\}$ and we put $\operatorname{split}(T)=\left\{s \in T:\left|\operatorname{succ}_{T}(s)\right| \geq 2\right\}$. For $s \in T, \operatorname{Succ}_{T}(s)=\{t \in$ $\left.\omega^{<\omega \uparrow}: s \frown t \in \operatorname{split}(T) \wedge \forall_{t^{\prime} \in \operatorname{split}(T)} s \subseteq t^{\prime} \subseteq s \frown t \Rightarrow\left(t^{\prime}=s \vee t^{\prime}=s \frown t\right)\right\}$. Finally, $[T]=\left\{x \in \omega^{\omega \uparrow}: \forall_{n \in \omega} x\lceil n \in T\}\right.$. We recall that a tree $T \subseteq \omega^{<\omega \uparrow}$ is said to be a Laver tree iff for every $s \in T$ with $\operatorname{stem}(T) \subseteq s, \operatorname{succ}_{T}(s)$ is infinite. A tree $T \subseteq \omega^{<\omega \uparrow}$ is a superperfect tree iff for any given $s \in T$, there is $t \supseteq s$ such that $\operatorname{succ}_{T}(t)$ is infinite. Let $[\omega]^{\omega}$ be the set of all infinite subsets of $\omega$. It is clear that an $x \in \omega^{\omega \uparrow}$ can be identified with an element of $[\omega]^{\omega}$ and vice versa. Thus, depending on the context, $\omega^{\omega \uparrow}$ is often conflated with $[\omega]^{\omega}$ or with the set $\left\{x: x \in 2^{\omega}\right.$ and $\left.\exists_{n}^{\infty} x(n)=1\right\}$.

Throughout this paper we assume that the measure used on $2^{\omega}$ is the product measure. By "+" we mean the usual modulo 2 coordinatewise addition in $2^{\omega}$ and for sets $A, B \subseteq 2^{\omega}$ we define $A+B=\{a+b: a \in A, b \in B\}$.

Let us recall that $X \subseteq 2^{\omega}$ is strongly meager iff for every measure zero set $A \subseteq 2^{\omega}$, there is $t \in 2^{\omega}$, so that $(X+t) \cap A=\emptyset$.

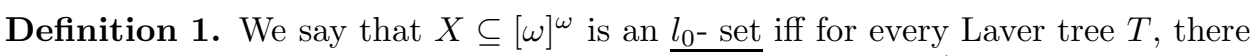
exists a Laver tree $S \subseteq T$ such that $\{\operatorname{ran}(x): x \in[S]\} \cap X=\emptyset$.

Definition 2. A set $X \subseteq[\omega]^{\omega}$ is called an $m_{0^{-}}$set iff for every superperfect tree $T$, there is a superperfect tree $S \subseteq T$ such that $\{\operatorname{ran}(x): x \in[S]\} \cap X=\emptyset$.

Received by the editors July 7, 2000 and, in revised form, October 2, 2000.

2000 Mathematics Subject Classification. Primary 03E15, 03E20, 28E15.

Key words and phrases. Strongly meager sets, Laver forcing, Miller forcing.

The first author was partially supported by KBN grant 2 P03A 04709. 
Theorem 1. Let $X \subseteq 2^{\omega}$ be a strongly meager set. Then $X$ has the $l_{0}$-property.

Proof. It suffices to show the following lemma.

Lemma 1. For every Laver tree $T \subseteq \omega^{<\omega \uparrow}$, there exists a measure zero set $H \subseteq 2^{\omega}$ such that

$\forall_{t \in 2^{\omega}} \exists_{S \subseteq T} S$ is a Laver tree \& $\operatorname{stem}(S)=\operatorname{stem}(T) \&\{\operatorname{ran}(x): x \in[S]\} \subseteq H+t$.

Proof. Before proving Lemma 1, we recall the following theorem due to Lorentz (see $[\mathrm{L}]$ ).

Fact 1. If $(G,+)$ is a finite group and if $H$ is a nonempty subset of $G$, then there is a subset $F$ of $G$ such that

1. The cardinality of $F$ is at most $|G| \cdot\left(\frac{1+\ln (|H|)}{|H|}\right)$, and

2. $G=F+H$.

To begin, we construct by induction a partition of $\omega$ into finite intervals as follows.

Let $I_{0}$ be equal to an interval $[0, n)$, which satisfies

$$
\max \left(I_{0}\right)>\max \operatorname{ran}(\operatorname{stem}(T)) .
$$

Having constructed $I_{0}, \ldots, I_{n}$ we define $I_{n+1}$, so that $\min \left(I_{n+1}\right)=\max \left(I_{n}\right)+1$ and for every $s \in T$ with the $\operatorname{stem}(T) \subseteq s, \max \operatorname{ran}(s) \leq \max \left(I_{n}\right)$,

$$
\left|\{k: \max \operatorname{ran}(s)<k \& s \frown\langle k\rangle \in T\} \cap I_{n+1}\right| \geq L_{n+1},
$$

where $L_{n+1}$ is any natural number satisfying

$$
\frac{\ln \left(L_{n+1}\right)+1}{L_{n+1}} \cdot 2^{\max I_{n}+1} \leq \frac{1}{2^{n+1}} .
$$

Now, for every $s \in T$ with the $\operatorname{stem}(T) \subseteq s, \max \operatorname{ran}(s) \leq \max \left(I_{n}\right)$, we apply Lorentz's theorem to get $\tilde{H}_{n+1}^{s} \subseteq 2^{I_{n+1}}$ such that:

1.

$$
\tilde{H}_{n+1}^{s}+\left\{e_{l}: l \in\{k: \max \operatorname{ran}(s)<k \& s \frown\langle k\rangle \in T\} \cap I_{n+1}\right\}=2^{I_{n+1}},
$$

2 .

$$
\left|\tilde{H}_{n+1}^{s}\right| \leq \frac{\ln \left(L_{n+1}\right)+1}{L_{n+1}} \cdot 2^{\left|I_{n+1}\right|},
$$

where $e_{l}$ for $l \in I_{n+1}$ is an element of $2^{I_{n+1}}$ given by

We define

$$
e_{l}(j)= \begin{cases}1 & \text { if } j=l \\ 0 & \text { if } j \neq l\end{cases}
$$

$$
H_{n+1}=\bigcup_{s \in T, \operatorname{stem}(T) \subseteq s, \max \operatorname{ran}(s) \leq \max \left(I_{n}\right)} \tilde{H}_{n+1}^{s}
$$

and we have

$$
\left|H_{n+1}\right| \leq \frac{\ln \left(L_{n+1}\right)+1}{L_{n+1}} \cdot 2^{\left|I_{n+1}\right|} \cdot 2^{\max I_{n}+1} \leq \frac{1}{2^{n+1}} \cdot 2^{\left|I_{n+1}\right|} .
$$

Thus,

$$
\sum_{n=0}^{\infty} \frac{\left|H_{n}\right|}{2^{\left|I_{n}\right|}}<\infty
$$


This implies that the set

$$
H=\left\{x \in 2^{\omega}: \exists_{n}^{\infty} x\left\lceil I_{n} \in H_{n}\right\}\right.
$$

is of measure zero.

We verify the following property:

$$
\forall_{t \in 2^{\omega}} \exists_{S \subseteq T} S \text { is a Laver tree \& }\{\operatorname{ran}(x): x \in[S]\} \subseteq H+t .
$$

Fix $t \in 2^{\omega}$. We construct a system $\left\{s_{r}\right\}_{r \in \omega<\omega}$ of elements from $\omega^{<\omega \uparrow}$ using induction on $|r|$. Suppose $s_{\emptyset}=\operatorname{stem}(T)$. Assume that we already have $\left\{s_{r}\right\}_{r \in \omega<n}$. Let $r \in \omega^{n-1}$. We choose $p_{r} \in \omega$ with $\max \operatorname{ran}\left(s_{r}\right)<\min \left(I_{p_{r}}\right)$. Then for every $p^{\prime} \in \omega$, there exists $k \in I_{p_{r}+p^{\prime}}$ satisfying:

1.

$$
\exists_{h \in H_{p_{r}+p^{\prime}}} e_{k}+h=t\left\lceil I_{p_{r}+p^{\prime}}\right.
$$

2.

$$
s_{r} \frown\langle k\rangle \in T .
$$

Let $s_{r \frown\left\langle p^{\prime}\right\rangle}=s_{r} \frown\langle k\rangle$, where $k$ is as above. Thus, for every $r \in \omega^{n-1}$ and $p^{\prime} \in \omega$, we have defined $s_{r \frown\left\langle p^{\prime}\right\rangle}$. Finally, we put

$$
S=\left\{s \in \omega^{<\omega \uparrow}: \exists_{r \in \omega<\omega} \subseteq \subseteq s_{r}\right\} .
$$

It is not hard to see that $S$ is a Laver tree, $S \subseteq T$, and $\operatorname{stem}(S)=\operatorname{stem}(T)$. Also, if $x \in[S]$, then $\exists_{n}^{\infty}\left(\left(t\left\lceil I_{n}\right)+\operatorname{ran}(x) \cap I_{n}\right) \in H_{n}\right.$, so we have

$$
\{\operatorname{ran}(x): x \in[S]\} \subseteq H+t .
$$

This completes our proof of Lemma 1 from which Theorem 1 easily follows.

Theorem 2. Let $X \subseteq 2^{\omega}$ be a strongly meager set. Then $X$ is an $m_{0}$-set.

Proof. As before it is sufficient to show the following lemma.

Lemma 2. For every superperfect tree $T \subseteq \omega^{<\omega \uparrow}$, there exists a measure zero set $H \subseteq 2^{\omega}$ such that

$$
\forall_{t \in 2^{\omega}} \exists_{S \subseteq T} S \text { is a superperfect tree \& } \operatorname{stem}(S)=\operatorname{stem}(T) \wedge
$$

$$
\{\operatorname{ran}(x): x \in[S]\} \subseteq H+t .
$$

Proof. Suppose we are given a superperfect tree $T \subseteq \omega^{<\omega \uparrow}$. Using the standard pruning argument we can assume that $\forall_{t \in T}\left|\operatorname{succ}_{T}(t)\right| \in\{1, \omega\}$.

Now we proceed as in the proof of Lemma 1 to get a sequence $\left\{I_{m}\right\}_{m \in \omega}$ of finite disjoint intervals in $\omega$ such that:

1.

$$
\min I_{m+1}=\max I_{m}+1
$$

2.

$$
\forall_{s \in \text { split }(T), \max \operatorname{ran}(s) \leq \max I_{m}}\left|\left\{t: t \in \operatorname{Succ}_{T}(s), \operatorname{ran}(t) \subseteq I_{m+1}\right\}\right| \geq L_{m+1},
$$

where $L_{m+1} \in \omega$ fulfills the following condition:

$$
\frac{\ln \left(L_{m+1}\right)+1}{L_{m+1}} \cdot 2^{\max I_{m}+1} \leq \frac{1}{2^{m+1}} .
$$


Let us denote

$$
H_{m+1}^{s}=\left\{t: t \in \operatorname{Succ}_{T}(s), \operatorname{ran}(t) \subseteq I_{m+1}\right\} .
$$

For every $s \in \operatorname{split}(T)$ with $\max \operatorname{ran}(s) \leq \max \left(I_{m}\right)$, we apply Lorentz's theorem to get $\tilde{H}_{m+1}^{s} \subseteq 2^{I_{m+1}}$ such that

$$
H_{m+1}^{s}+\left\{t: t \in \tilde{H}_{m+1}^{s}\right\}=2^{I_{m+1}} .
$$

We define

$$
H_{m+1}=\bigcup_{\max \operatorname{ran}(s) \leq \max \left(I_{m}\right)} \tilde{H}_{m+1}^{s} .
$$

This completes the recursive construction of the sequence $\left\{H_{m}\right\}_{m \in \omega}$ and we argue as before to get a measure zero set $H$.

Let $t \in 2^{\omega}$. We construct, by induction on $|r|$ a sequence $\left\{s_{r}\right\}_{r \in \omega<\omega \uparrow}$, so that $s_{r} \in \operatorname{split}(T)$. So assume that we already have $\left\{s_{r}\right\}_{r \in \omega^{m-1}}$. First choose $p_{r} \in \omega$ such that $\max \left(s_{r}\right)<\min \left(I_{p_{r}}\right)$. Then for every $p^{\prime} \in \omega$, there is $\tilde{t}$, with $\operatorname{ran}(\tilde{t}) \subseteq I_{p_{r}+p^{\prime}}$, which satisfies

1 .

$$
\exists_{h \in H_{p_{r}+p^{\prime}}} \tilde{t}+h=t\left\lceil I_{p_{r}+p^{\prime}},\right.
$$

2.

$$
s_{r} \frown \tilde{t} \in T .
$$

We put $s_{r \frown p^{\prime}}=s_{r} \frown \tilde{t}$, where $\tilde{t}$ is as above and we set

$$
S=\left\{s \in \omega^{<\omega \uparrow}: \exists_{r \in \omega<\omega \uparrow} \subseteq s_{r}\right\} .
$$

Let $x \in[S]$. Clearly, $\exists_{n}^{\infty}\left(\left(t\left\lceil I_{n}\right)+\operatorname{ran}(x) \cap I_{n}\right) \in H_{n}\right.$.

Remark. We shall say that an $X \subseteq 2^{\omega}$ is a very meager (VFC) set iff for every $H \subseteq$ $2^{\omega}$ of measure zero there are sequences $\left\{X_{n}\right\}_{n \in \omega},\left\{t_{n}\right\}_{n \in \omega}$ such that $X \subseteq \bigcup_{n \in \omega} X_{n}$ and $t_{n} \in X_{n}+H$, for $n \in \omega$. It is easy to modify the proof of Theorem 4 from NW to show that sets with the latter property are $C R_{0}$. Also one can check that Theorem 1 and Theorem 2 of this paper remain true with a strongly meager set replaced by a VFC set. To see this, let $\left\{A_{n}\right\}_{n \in \omega}$ be a partition of $\omega$ into disjoint infinite sets and assume that $\left\{t_{n}\right\}_{n \in \omega}$ is a sequence of reals. We construct a system $\left\{s_{r}\right\}_{r \in \omega<\omega}$ of elements from $\omega^{<\omega \uparrow}$ by induction on $|r|$ using the same method as in the proof of Lemma 1$]$ with condition 1 replaced by the following requirement. If $\left\{s_{r}\right\}_{r \in \omega<n}$ are already constructed and $n \in A_{m}$ for some $m \in \omega$, then we choose $p_{r} \in \omega$ satisfying $\max \operatorname{ran}\left(s_{r}\right)<\min \left(I_{p_{r}}\right)$ and for every $p^{\prime} \in \omega$ we pick $k \in I_{p_{r}+p^{\prime}}$, so that:

1.

$$
\exists_{h \in H_{p_{r}+p^{\prime}}} e_{k}+h=t_{m}\left\lceil I_{p_{r}+p^{\prime}},\right.
$$

2 .

$$
s_{r} \frown\langle k\rangle \in T \text {. }
$$

Clearly, we will have that $\{\operatorname{ran}(x): x \in[S]\} \subseteq H+t_{m}$, for every $m \in \omega$, where $S=\left\{s \in \omega^{<\omega \uparrow}: \exists_{r \in \omega}<\omega s \subseteq s_{r}\right\}$. Thus if we assume that $X \subseteq \bigcup_{m \in \omega} X_{m}$ and $X_{m} \cap\left(H+t_{m}\right)=\emptyset$, for every $m \in \omega$, then $\{\operatorname{ran}(x): x \in[S]\} \cap X=\emptyset$. 


\section{ADDED AFTER POSTING}

It was noticed by M. Kysiak that Theorem 2 holds for a wider class of sets, namely perfectly meager subsets of $2^{\omega}$. Let us notice also that the definition of VFC sets is due to M. Kysiak.

\section{REFERENCES}

[B] J. Brendle, Strolling through paradise, Fundamenta Mathematicae 148 (1995), 1 - 25. MR 96h:03085

[L] G.G. Lorentz, On a problem of additive number theory, Proceedings of the American Mathematical Society 5 (1954), $838-841$. MR 16:113f

[NW] A. Nowik, T. Weiss, Remarks on strongly meager sets of real numbers and their uniformly continuous images, Proceedings of the AMS. 129 (2001), 265 - 270. MR 2001c:03081

University of Gdańsk, Institute of Mathematics, ul. Wita Stwosza 57, 80-952 Gdańsk, POLAND

E-mail address: matan@paula.univ.gda.pl

Current address: Institute of Mathematics, Polish Academy of Sciences, Abrahama 18, 81-825 Sopot, Poland

Institute of Mathematics, WSRP, 08-110 Siedlce, Poland

E-mail address: weiss@wsrp.siedlce.pl 